\title{
O uso de testes de fluência verbal como ferramenta de rastreio cognitivo em idosos
}

\author{
Alina L. G. Teldeschi, ${ }^{1, \star}$ Mariangela Perez, ${ }^{1}$ Maria Angélica Sanchez, ${ }^{1}$ Roberto A. Lourenço ${ }^{2}$
}

\section{Resumo}

Introdução: Instrumentos de avaliação capazes de identificar quadros demenciais são fundamentais na prática clínica e de pesquisa. O teste de fluência verbal é um instrumento de avaliação cognitiva breve que vem mostrando alto potencial. Objetivos: Verificar estudos publicados sobre desempenho de idosos com e sem comprometimento cognitivo em testes de fluência verbal (TFV). Métodos: Revisão narrativa em bases de dados eletrônicas de artigos originais e de revisão que utilizaram o teste de fluência verbal, categoria animais. Resultados e Discussão: Foram descritos estudos que investigaram o desempenho de idosos em TFV nas síndromes demenciais de maior prevalência. Na doença de Alzheimer, o desempenho no TFV está comprometido desde o início do curso da doença, sendo mais expressivo o prejuízo em testes de fluência verbal com critério semântico (TFVCS). Nas demências vasculares, o comprometimento em tarefas que demandam funções executivas, atenção e velocidade de processamento está associado ao pior desempenho em TFV com critério ortográfico. Na demência por corpos de Lewy (DCL), o desempenho nos TFV critério semânticoé semelhante aos de indivíduos com DA e ao de indivíduos com doença de Parkinson(DP). Nos testes com critério ortográfico, o desempenhoé semelhante ao de indivíduos com DP e inferior ao de indivíduos com DA. Conclusões: Os TFVs são sensíveis à presença de alterações cognitivas em idosos e, portanto, podem contribuir para a identificação de casos de demência em ambientes de escassez de recursos.

Descritores: Demência; Testes neuropsicológicos; Idoso.

\section{Abstract \\ The use of verbal fluency tests as a cognitive scre- ening tool in the elderly}

Introduction: Evaluation tools capable of contributing effectively to the identification of potential dementia cases are fundamental in clinical practice and research. The verbal fluency test is a brief cognitive test that has shown high potential in this direction. Objectives: Considering the wide use of these tests in clinical and research context, the present article has proposed to survey studies that investigated the performance of older adults with and without cognitive impairment in verbal fluency tests (VFT). Methods: It is a narrative/critical review with a search in electronic databases for original and review articles about verbal fluency tests using animal category. Results and Discussion: The article found studies investigating the performance of old people in the VFT in the most prevalent dementia syndromes. In the Alzheimer's disease (AD), the VFT performance is affected since
1. Laboratório de Pesquisa em Envelhecimento Humano-GeronLab. Universidade do Estado do Rio de Janeiro. Rio de Janeiro, RJ, Brasil.

2. Departamento de Medicina Interna. Hospital Universitário Pedro Ernesto. Universidade do Estado do Rio de Janeiro. Rio de Janeiro, RJ, Brasil.

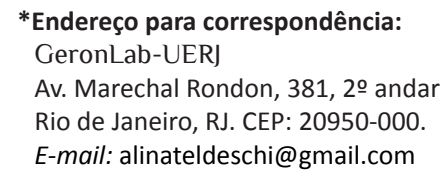

the beginning of the disease, with a major negative injury in the semantic verbal fluency tests. In the vascular dementias, the impairment in tasks that demand executive function, attention, processing speed is associated to the worse performance in the VTF letter category. In the dementia with Lewy bodies (LBD), the performance in the semantic verbal fluency tests is similar to the performance of individuals with AD and those with Parkinson's disease (PD). In the VTF letter category, the performance is similar to that of individuals with PD, and worse than that of individuals with AD. Conclusions: VFT are sensible to the cognitive alterations in the elderly, thus, they can actually contribute to the screening of dementia in situations of scarce resources.

Keywords: Dementia; Neuropsychological tests; Aged.

\section{Resumen}

El uso de pruebas de fluidez verbal como herramienta de evaluación cognitiva en adultos mayores

Los instrumentos de valorización capaces de contribuir de manera eficaz en la identificación de potenciales cuadros demenciales son fundamentales en la práctica clínica y en la investigación. La prueba de fluidez verbal es un instrumento de valorización cognitiva breve que viene mostrando alto potencial en esta dirección. Objetivos: Considerando el amplio uso de estas pruebas en el ámbito clínico y de investigación, el presente artículo se propuso hacer un levantamiento de estudios que investigaron el desempeño de ancianos con y sin compromiso cognitivo en pruebas de fluidez verbal. Métodos; El artículo es una revisión narrativa/crítica con búsqueda en bases de datos electrónicas de artículos originales y de revisión 
sobre pruebas de fluidez verbal, utilizando categorías de animales. Resultados y Discusión: El artículo encontró estudios que investigan el desempeño de las personas mayores en la prueba de fluidez verbal (PFV) en los sindromes de demencia mas prevalentes. En la enfermedad de Alzheimer (EA), el rendimiento de la PFV se ve afectada desde el comienzo de la enfermedad, con una lesión negativa importante en las pruebas de fluidez verbal semántica. En las demencias vasculares, el deterioro en las tareas que demandan la función ejecutiva, la atención, la velocidad de procesamiento está asociada al peor desempeño en la categoría de letras (PFV).En la demencia

\section{A identificação de casos de demência}

Oenvelhecimento populacional vem acompanhado de um aumento da prevalência de doenças crônicas e incapacitantes, dentre as quais se destaca a demência, uma síndrome orgânica cerebral, caracterizada pelo comprometimento progressivo de processos cognitivos como memória, atenção, orientação, linguagem, julgamento, funções executivas, praxia, entre outros. Tais perdas geram uma série de prejuízos pessoais, sociais e ocupacionais, sobretudo o prejuízo da capacidade de realizar atividades de vida diária de maneira independente. ${ }^{1}$

Apesar de dolorosa, a confirmação de um diagnóstico de demência traz muitos benefícios para o paciente, seus familiares e cuidadores. Ela permite uma melhor compreensão das mudanças comportamentais observadas e favorece a busca de orientações acerca dos cuidados que serão necessários no dia a dia, para que se possa garantir a segurança e a qualidade de vida do indivíduo acometido pela doença. A partir deste ponto, os familiares e cuidadores poderão também buscar profissionais da área da saúde que os ajudem a lidar com os sintomas de modo menos desgastante. ${ }^{2}$

A identificação de casos de demência é uma questão bastante complexa, principalmente em países de média e baixa renda, onde o acesso à rede de proteção social e aos serviços de saúde e educação é mais limitado. ${ }^{3}$ Nestes países há um número elevado de indivíduos com demência que nunca receberam diagnóstico. ${ }^{4} \mathrm{No}$ Brasil, estima-se que $77 \%$ das pessoas com demência não tenham um diagnóstico firmado. ${ }^{5}$

Para que um número maior de indivíduos com demência possa ser identificado, é fundamental a participação dos profissionais da atenção primária e a cooperação destes com os profissionais da atenção secundária. Assim, o processo de investigação diagnóstica ocorreria em duas etapas. Na primeira etapa os idosos con cuerpos de Lewy, el rendimiento en las pruebas de fluidez verbal semántica es similar al rendimiento de las personas con la enfermedad de Alzheimer y de aquellos con la enfermedad de Parkinson (EP). En la categoría de letras PFV, el rendimiento es similar al de las personas con EP, y peor que el de las personas con EA. Conclusiones: Las pruebas de fluidez verbal (PFV) son sensibles a las alteraciones cognitivas en los ancianos, por lo tanto, pueden contribuir a la detección de la demencia en situaciones de escasos recursos.

Palabras clave: Demencia; Pruebas neuropsicológicas; Anciano.

passariam por uma triagem que investigaria a presença de comprometimento cognitivo. ${ }^{6}$

Como na atenção primária, a disponibilidade de tempo e recursos é baixa, o uso de um teste cognitivo breve com boas medidas de validade aparece como uma alternativa bastante útil. Embora o uso destes instrumentos não seja suficiente para o estabelecimento do diagnóstico de demência, eles auxiliam na identificação daqueles indivíduos que necessitam ser encaminhados para um serviço de avaliação cognitiva especializado. ${ }^{1}$

\section{O uso de testes cognitivos breves na investigação de síndrome demencial}

Alguns autores defendem a inclusão do rastreamento cognitivo na rotina de cuidados de saúde dos idosos, especialmente para aqueles que apresentam fatores de risco para demência, como, por exemplo, idade maior ou igual a 85 anos., ${ }^{7,8}$ Outros autores recomendam que o rastreio cognitivo seja realizado assim que surgir uma preocupação em relação à capacidade cognitiva ou quando forem percebidas dificuldades na execução de atividades de vida diária, como, por exemplo, administração financeira. ${ }^{9}$

Diferentes estudos trazem evidências que dão suporte a estas recomendações. Eichler e colaboradores revelaram que um número considerável de pacientes com comprometimento cognitivo (30\% da amostra) só foi reconhecido pelos médicos generalistas após o uso de um teste de rastreio..$^{10}$ Borson e colaboradores mostraram que quando um médico generalista usa um teste de rastreio cognitivo, as chances de se detectar uma síndrome demencial são muito maiores do que quando não o utilizam ( $83 \%$ versus 59\%, respectivamente). ${ }^{11}$

Existem inúmeros testes cognitivos disponíveis para uso clínico que possuem as vantagens de serem breves, de baixo ou nenhum custo, fáceis de serem 


\section{Artigo de revisão}

administrados e com boa acurácia. A tabela 1 apresenta instrumentos de rastreio validados para a população brasileira.

No entanto, apesar das vantagens subjacentes aos testes de rastreio cognitivo, cabe ressaltar o fato de que não existe um único teste $100 \%$ eficaz. A escolha do instrumento deve levar em consideração o contexto onde será utilizado e o perfil clínico e sociodemográfico da população a ser atendida, além de, preferencialmente, possuir normas condizentes com a idade e a escolaridade do indivíduo que está sendo avaliado.

Em uma clínica de atenção primária muito atribulada, por exemplo, pode ser impraticável aplicar o Miniexame do Estado Mental (MEEM), cujo tempo de aplicação varia em torno de 10 minutos. ${ }^{20}$ Neste caso, um teste mais breve, como o teste de fluência verbal, que dura cerca de 2 minutos, pode ser uma alternativa mais viável. ${ }^{21}$

\section{Teste de fluência verbal}

Genericamente, testes de fluência verbal (TFV) são aqueles que requerem geração espontânea do maior número de palavras possível, dentro de um limite de tempo (em geral, 1 minuto), de acordo com critérios semânticos (palavras pertencentes a uma determinada categoria) e/ou ortográficos (palavras que se iniciam por determinada letra). ${ }^{21}$

\section{O desempenho de indivíduos com demência em testes de fluência verbal}

O TFV é muito utilizado na investigação do funcionamento cognitivo de idosos. ${ }^{21,22} \mathrm{O}$ desempenho deficitário em TFV pode ser um sinalizador de dificuldades funcionais. Uma pesquisa realizada por Cahn-Weiner, Boyle e Malloy mostrou que o desempenho nestes testes contribuía de maneira significativa e independente para a predição de escores em uma escala de Atividades Instrumentais de Vida Diária preenchida por cuidadores de idosos de uma comunidade. ${ }^{23}$

A seguir serão abordados estudos que investigaram o desempenho de idosos em TFV nas síndromes demenciais com maior prevalência.

\section{Doença de Alzheimer}

Indivíduos com doença de Alzheimer (DA) costumam apresentar desempenho comprometido em TFV desde o início do curso da doença. ${ }^{24} \mathrm{O}$ prejuízo observado em testes de fluência verbal com critério semântico (TFVCS) 21,22 é o mais expressivo.

Estudos que compararam o desempenho de indivíduos com DA em TFVCS e em testes de fluência verbal com critério ortográfico (TFVCO) mostraram

Tabela 1. Instrumentos de rastreio cognitivo em idosos validados para a população brasileira

\begin{tabular}{|c|c|c|}
\hline Instrumento & O que avalia & Duração aproximada \\
\hline Miniexame do Estado Mental ${ }^{12}$ & $\begin{array}{l}\text { Orientação temporal e espacial, memória de curto prazo, cálculo, } \\
\text { linguagem, visuoconstrução e habilidades visuoespaciais }\end{array}$ & 10 minutos \\
\hline $\begin{array}{l}\text { Montreal Cognitive Assessment } \\
(\mathrm{MoCa})^{13}\end{array}$ & $\begin{array}{l}\text { Funções executivas, habilidades visuoespaciais, memória de curto } \\
\text { prazo, atenção, memória operacional, linguagem e orientação }\end{array}$ & 10 minutos \\
\hline Teste do Desenho do Relógio ${ }^{14}$ & Habilidades visuoespaciais, visuoconstrução e funções executivas & 1-2 minutos \\
\hline Mini-Cog ${ }^{15}$ & $\begin{array}{l}\text { Memória de curto prazo, habilidades visuoespaciais, } \\
\text { visuoconstrução e funções executivas }\end{array}$ & 3 minutos \\
\hline Bateria Breve de Rastreio Cognitivo ${ }^{16}$ & $\begin{array}{l}\text { Nomeação, memória incidental, memória imediata, aprendizado, } \\
\text { resgate tardio e reconhecimento, fluência verbal, habilidades } \\
\text { visuoespaciais, visuoconstrução e funções executivas }\end{array}$ & 6-7 minutos \\
\hline $\begin{array}{l}\text { Exame Cognitivo de Addenbrooke- } \\
\text { Revisado (Addenbrooke's Cognitive } \\
\text { Examination-Revised: ACE-R) }{ }^{17}\end{array}$ & $\begin{array}{l}\text { Orientação, atenção, memória, fluência verbal, linguagem e } \\
\text { habilidades visuoespaciais }\end{array}$ & 15-20 minutos \\
\hline $\begin{array}{l}\text { Cognitive Abilities Screening } \\
\text { Instrument Short Form (CASI-S) }{ }^{18}\end{array}$ & Memória de curto prazo, orientação temporal e fluência verbal & 4 minutos \\
\hline Teste de fluência verbal ${ }^{19}$ & Linguagem e funções executivas & 1-3 minutos \\
\hline
\end{tabular}


que a capacidade de gerar palavras de acordo com um critério semântico declina de forma mais acelerada e acentuada ao longo do curso da doença. ${ }^{22,25}$ Os TFVCS acabam, portanto, apresentando melhores medidas de sensibilidade e especificidade do que os TFVCO, na diferenciação de indivíduos com DA daqueles sem a doença. ${ }^{26}$

A perda da capacidade de evocar palavras de acordo com um critério semântico está diretamente associada à deterioração das redes neurais acometidas pela DA. Estudos histopatológicos e de neuroimagem funcional realizados em indivíduos com DA mostram que algumas das regiões cerebrais mais acometidas ao longo do curso da doença estão associadas ao sistema semântico, como os córtices de associação temporal, frontal e parietal. 25,26

A deterioração do sistema semântico é caracterizada pelo enfraquecimento das associações entre conceitos semanticamente relacionados e pela perda de conceitos mais específicos/menos generalistas (ex. laranja lima). O conhecimento semântico mais generalista (conceitos que abrangem muitas coisas, por exemplo a palavra 'objeto' pode se referir a diferentes itens no ambiente) é o último a ser afetado na linha de progressão da DA. ${ }^{25,27}$

Martin e Fedio constataram este perfil de desempenho em um teste de fluência verbal que solicitava a evocação de itens pertencentes a um supermercado. Os autores observaram que os indivíduos com DA falavam poucos itens específicos (ex. cenouras) e tendiam a evocar palavras mais generalistas, como frutas e vegetais. Em contraposição, os indivíduos sem demência conseguiam realizar uma busca por conceitos de maneira mais sistemática/ordenada e falar um maior número de conceitos específicos. ${ }^{27}$

\section{Demência vascular}

A demência vascular (DV) é uma condição caracterizada por comprometimento cognitivo e funcional associado a lesões cerebrais diversas de origem vascular/ circulatória. Ela pode se apresentar de diferentes maneiras, dependendo do tipo de alteração cerebrovascular e da localização e extensão da lesão. A diversidade de manifestações faz com que as alterações cognitivas secundárias à demência vascular sejam muito mais variáveis do que as observadas em outras demências. ${ }^{28}$ Esta variabilidade é refletida em estudos que investigaram o desempenho de indivíduos com DV em TFV.

Starkstein e colaboradores mostraram que o maior acometimento de regiões fronto-subcorticais em indivíduos com DV em relação a indivíduos com DA estava associado a um pior desempenho em tarefas que demandam funções executivas, atenção e velocidade de processamento, como os TFVCO. ${ }^{29}$

Um estudo longitudinal de 12 meses de acompanhamento que avaliou indivíduos com DV mostrou que pontuações mais baixas na escala de Hachinski, menor volume cerebral total e maior quantidade de hiperintensidades de substância branca, na ressonância nuclear magnética (RNM) do crânio, estavam associados a um pior desempenho no TFV com as letras F-A-S.30

Lafosse e colaboradores também encontraram associação entre a magnitude/gravidade das alterações em substância branca ("leukaraiosis"), na RNM, e o desempenho em TFVCO. ${ }^{31}$

\section{Demência por corpos de Lewy}

A demência por corpos de Lewy (DCL) é uma doença neurodegenerativa associada a sintomas extrapiramidais, alucinações visuais, distúrbios do sono, comprometimento cognitivo e flutuação do estado de consciência. 32,33

O prejuízo cognitivo desta condição é caracterizado por deficitem atenção, funções executivas e habilidades visuoespaciais, com menores dificuldades de memória nos estágios iniciais. ${ }^{33} \mathrm{O}$ declínio destas funções comumente compromete o desempenho de indivíduos com DCL em TFV. ${ }^{34}$

Em TFVCS, os indivíduos com DCL costumam apresentar desempenho semelhante ao de indivíduos com $\mathrm{DA}^{35,36} \mathrm{e}$ com demência relacionada à doença de Parkinson. ${ }^{37}$ Em TFVCO, o desempenho de indivíduos com DCL costuma ser semelhante ao de indivíduos com demência relacionada à doença de Parkinson e significativamente inferior ao de indivíduos com DA. . $5,36,38^{2}$

\section{Considerações finais}

Os TFV têm as vantagens de serem breves, de baixo ou nenhum custo e fáceis de administrar, podendo ser utilizados por qualquer profissional treinado. Os estudos apresentados nesta revisão mostram que este tipo de teste costuma ser sensível à presença de alterações cognitivas em idosos e que, portanto, podem contribuir bastante para a identificação de casos de demência em contextos caracterizados pela escassez de tempo e recursos.

Ressalta-se que o desempenho em TFV deve ser interpretado levando em consideração a idade e escolaridade do examinando, pois estas variáveis, em geral, possuem correlação com os resultados obtidos 


\section{Artigo de revisão}

\section{em testes cognitivos.}

\section{Referências}

1. Slater $\mathrm{H}$, Young J. A review of brief cognitive assessment tests. Rev Clin Gerontol. 2013;23:164-176.

2. Borson S, Frank L, Bayley PJ, et al. Improving dementia care: The role of screening and detection of cognitive impairment. Alzheimers Dement. 2013;9:151-159.

3. Prince M, Wimo A, Guerchet M, et al. World Alzheimer Report 2015 - The Global Impact of Dementia: An analysis of prevalence, incidence, cost and trends. Alzheimer's Disease International. 2015.

4. Alzheimer's Disease International. Policy Brief for Heads of Government. The Global Impact of Dementia 2013-2050. 2013.

5. Nakamura AE, Opaleye D, Tani G, et al. Dementia underdiagnosis in Brazil. Lancet. 2015;385:418-419.

6. llife S, Robinson L, Brayne C, et al. Primary care and dementia: 1. diagnosis, screening and disclosure. Int J Geriatr Psychiatry. 2009;24:895-901.

7. Ashford JW, Borson S, O'Hara R, et al. Should older adults be screened for dementia? It is important to screen for evidence of dementia! Alzheimers Dement. 2007;3:75-80.

8. Tai S, Huang SW, Hsu CL, et al. Screening Dementia in the Outpatient Department: Patients at Risk for Dementia. Scientific World Journal. 2014;2014:138786.

9. Panegyres PK, Berry R, Burchell J. Early Dementia Screening. Diagnostics (Basel). 2016;6, pii: E6

10. Eichler T, Thyrian JR, Hertel J, et al. Rates of formal diagnosis of dementia in primary care: The effect of screening. Alzheimers Dement (Amst). 2015;1:87-93.

11. Borson S, Scanlan JM, Watanabe J, et al. Improving identification of cognitive impairment in primary care. Int $\mathrm{J}$ Geriatr Psychiatry. 2006;21:349-55.

12. Melo DM de, Barbosa AJG. O uso do Mini-Exame do Estado Mental em pesquisas com idosos no Brasil: uma revisão sistemática. Ciênc. Saúde Coletiva [online]. 2015;20:3865-3876.

13. Sarmento, ALR. Apresentação e aplicabilidade da versão brasileira da MoCA (Montreal Cognitive Assessment) para rastreio de Comprometimento Cognitivo Leve [dissertação de mestrado]. [São Paulo]: Universidade Federal de São PauloEscola Paulista de Medicina (UNIFESP); 2009. 82 p.

14. Lourenço RA, Filho STR, Moreira IFH, et al. Teste do desenho do relógio: Desempenho entre idosos de baixa-escolaridade. Rev Bras Psiquiatr. 2008;30:309-315.

15. Filho STR, Lourenço RA. The performance of the Mini-Cog in a sample of low educational level elderly. Dement Neuropsychol. 2009;3:81-87.

16. Nitrini R, Caramelli P, Porto CS, et al. Brief cognitive battery in the diagnosis of mild Alzheimer's disease in subjects with medium and high levels of education. Dement Neuropsychol. 2007;1:32-36.

17. Carvalho VA, Caramelli P. Brazilian adaptation of the Addenbrooke's Cognitive Examination-Revised (ACE-R). Dement Neuropsychol. 2007;1:212-216.

18. Damasceno A, Delicio AM, Mazo DFC, et al. Validation of the Brazilian version of mini-test CASI-S. Arq. Neuro-Psiquiatr. [online]. 2005;63:416-421.

19. Teldeschi ALG. Acurácia dos testes de fluência verbal com critério semântico e ortográfico na identificação de idosos com síndrome demencial: estudo FIBRA - RJ [dissertação de mestrado]. [Rio de Janeiro]: Universidade do Estado do Rio de Janeiro; 2017.106 p.
20. Kilada S, Gamaldo A, Grant EA, et al. Brief Screening Tests for the Diagnosis of Dementia: Comparison With the Mini-Mental State Exam. Alzheimer Dis Assoc Disord. 2005;19:8-16

21. Canning SJD, Leach L, Stuss D, et al. Diagnostic utility of abbreviated fluency measures in Alzheimer disease and vascular dementia. Neurology. 2004;62:556-62.

22. Clark LJ, Gatz M, Zheng L, et al. Longitudinal Verbal Fluency in Normal Aging, Preclinical, and Prevalent Alzheimer's Disease. Am J Alzheimers Dis Other Demen. 2010;24:461-8.

23. Cahn-Weiner DA, Boyle PA, Malloy PF. Tests of Executive Function Predict Instrumental Activities of Daily Living in Community-Dwelling Older Individuals. Appl Neuropsychol. 2002;9:187-91.

24. Amieva H, Le Goff M, Millet X, et al. Prodromal Alzheimer's Disease: Successive Emergence of the Clinical Symptoms. Ann Neurol. 2008;64:492-8.

25. Salmon DP, Heindel WC, Lange KL. Differential decline in word generation from phonemic and semantic categories during the course of Alzheimer's disease: Implications for the integrity of semantic memory. J Int Neuropsychol Soc. 1999;5:692-703.

26. Monsch AU, Bondi MW, Butters N, et al. Comparisons of Verbal Fluency Tasks in the Dementia of the Alzheimer Type. Arch Neurol. 1992;49:1253-8.

27. Martin A, Fedio P. Word production and comprehension in Alzheimer's disease: The breakdown of semantic knowledge. Brain Lang. 1983;19:124-41.

28. O'Brien JT, Thomas A. Vascular dementia. Lancet. 2015;386:1698-706.

29. Starkstein SE, Sabe L, Vazquez S, et al. Neuropsychological, Psychiatric, and Cerebral Blood Flow Findings in Vascular Dementia and Alzheimer's Disease. Stroke. 1996; 27:408-14.

30. Paul RH, Cohen RA, Moser DJ, et al. Clinical Correlates of Cognitive Decline in Vascular Dementia. Cogn Behav Neurol. 2003;16:40-6.

31. Lafosse JM, Reed BR, Mungas D, et al. Fluency and Memory Differences Between Ischemic Vascular Dementia and Alzheimer's Disease. Neuropsychology. 1997;11:514-22.

32. Aarsland D. Cognitive impairment in Parkinson's disease and dementia with Lewy Bodies. Parkinsonism Relat Disord. 2016; Suppl 1:S144-8.

33. Collerton D, Burn D, McKeith I, et al. Systematic Review and Meta-Analysis Show that Dementia with Lewy Bodies is a Visual-Perceptual and Attentional-Executive Dementia. Dement Geriatr Cogn Disord. 2003;16:229-37.

34. Crowell TA, Luis CA, Cox DE, et al. Neuropsychological Comparison of Alzheimer's Disease and Dementia with Lewy Bodies. Dement Geriatr Cogn Disord. 2007;23:120-5.

35. Nervi A, Reitz C, Tang MX, et al. Comparison of clinical manifestations in Alzheimer disease and dementia with Lewy bodies. Arch Neurol. 2008;65:1634-9.

36. Calderon J, Perry RJ, Erzinclioglu SW, et al. Perception, attention, and working memory are disproportionately impaired in dementia with Lewy bodies compared with Alzheimer's disease. J Neurol Neurosurg Psychiatry. 2001;70:157-64.

37. Petrova M, Mehrabian-Spasova S, Aarsland D, et al. Clinical and Neuropsychological Differences between Mild Parkinson's Disease Dementia and Dementia with Lewy Bodies. Dement Geriatr Cogn Dis Extra. 2015;5:212-20.

38. Salmon DP, Bondi MW. Neuropsychological Assessment of Dementia. Annu Rev Psychol. 2009;60:257-82. 